\title{
The Exponentiated Gumbel Type-2 Distribution: Properties and Application
}

\author{
I. E. Okorie, ${ }^{1}$ A. C. Akpanta, ${ }^{2}$ and J. Ohakwe ${ }^{3}$ \\ ${ }^{1}$ School of Mathematics, University of Manchester, Manchester M13 9PL, UK \\ ${ }^{2}$ Department of Statistics, Abia State University, PMB 2000, Uturu, Abia State, Nigeria \\ ${ }^{3}$ Department of Mathematics \& Statistics, Faculty of Sciences, Federal University, Otuoke, PMB 126, Yenagoa, Bayelsa, Nigeria
}

Correspondence should be addressed to I. E. Okorie; idika.okorie@postgrad.manchester.ac.uk

Received 17 May 2016; Accepted 10 July 2016

Academic Editor: Niansheng Tang

Copyright (C) 2016 I. E. Okorie et al. This is an open access article distributed under the Creative Commons Attribution License, which permits unrestricted use, distribution, and reproduction in any medium, provided the original work is properly cited.

\begin{abstract}
We introduce a generalized version of the standard Gumble type-2 distribution. The new lifetime distribution is called the Exponentiated Gumbel (EG) type-2 distribution. The EG type-2 distribution has three nested submodels, namely, the Gumbel type2 distribution, the Exponentiated Fréchet (EF) distribution, and the Fréchet distribution. Some statistical and reliability properties of the new distribution were given and the method of maximum likelihood estimates was proposed for estimating the model parameters. The usefulness and flexibility of the Exponentiated Gumbel (EG) type-2 distribution were illustrated with a real lifetime data set. Results based on the log-likelihood and information statistics values showed that the EG type- 2 distribution provides a better fit to the data than the other competing distributions. Also, the consistency of the parameters of the new distribution was demonstrated through a simulation study. The EG type-2 distribution is therefore recommended for effective modelling of lifetime data.
\end{abstract}

\section{Introduction}

The Gumbel distribution, also known as the type-1 extreme value distribution, has received significant research attention, over the years particularly, in extreme value analysis of extreme events. For a review of the recent developments and applications of the Gumbel distribution, see Pinheiro and Ferrari [1]. There is no question that, before now, the Gumbel type-2 distribution is not popularly used in statistical modelling and the reason may not be far from its lack of fits in data modelling. Generally, standard probability distributions are well known for their lack of fits in modelling complex data sets. On this note, users of this distributions across various fields in general and statistics and mathematics in particular have been fantastically motivated to developing sophisticated probability distributions from the existing ones. Exponentiated distributions have been introduced to solve the problem of lack of fits that is commonly encountered when using the standard probability distributions for modelling complex data sets. Results from this advancement have frequently been proven more reasonable than the one based on the standard distributions. Exponentiating distributions are indeed a powerful technique in statistical modelling that offers an effective way of introducing an additional shape parameter to the standard distribution to achieve robustness and flexibility. This method of generalizing probability distributions is traceable to the work of Gupta et al. [2] who introduced the exponentiated exponential (EE) distribution as a generalized form of the standard exponential distribution by simply raising the cumulative density function (cdf) to a positive constant power. Ever since the introduction of the EE distribution, exponentiated distributions have achieved reasonable feats in modelling data sets from various complex phenomena. A good number of standard probability distributions have their corresponding exponentiated versions. Gupta et al. [2] introduced the Exponentiated Weibull distribution as a generalization of the standard Weibull distribution. Nadarajah and Kotz [3] modified the method by Gupta et al. [2] and introduced the Exponentiated Fréchet distribution as a generalization of the standard Fréchet distribution. Using the same method in Nadarajah and Kotz [3], Nadarajah [4] introduced the 
Exponentiated Gumbel distribution as a generalization of the standard Gumbel distribution. Mudholkar and Srivastava [5] introduced the Exponentiated Weibull family distribution as a generalization of the Weibull family distribution. Ashour and Eltehiwy [6] developed the exponentiated power Lindley distribution generalizing the power Lindley distribution and so forth. Therefore, this paper is aimed at generalizing the standard Gumbel type-2 distribution to a wider class of distribution so as to improve its performance and encourage its applicability, in modelling varieties of complex data sets.

The cumulative density function $\operatorname{cdf}(F(x))$ of the exponentiated family of distributions according to Nadarajah and Kotz [3] is defined by

$$
\begin{aligned}
& F(x ; \underline{\omega} ; \alpha)=1-(1-G(x ; \underline{\omega}))^{\alpha} \\
& \\
& x \in \mathfrak{R} ; \alpha>0 ; \underline{\omega} \in \Omega ;
\end{aligned}
$$

differentiating (1) with respect to $x$ gives the corresponding probability density function $\operatorname{pdf}(f(x))$ as

$$
\begin{aligned}
& f(x ; \underline{\omega} ; \alpha)=\alpha g(x ; \underline{\omega})(1-G(x ; \underline{\omega}))^{\alpha-1} ; \\
& x \in \mathfrak{R} ; \alpha>0 ; \underline{\omega} \in \Omega,
\end{aligned}
$$

where $\underline{\omega}$ and $\Omega$ are the vector of parameters and parameter space of the baseline distribution $(G(x ; \underline{\omega}))$, respectively.

The remaining part of this paper is organized as follows; Section 2 introduces the Gumbel type- 2 distribution, its exponentiated version, and special cases (submodels); Section 3 presents some important reliability characteristics of the new distribution and their asymptotic properties; Section 4 presents an explicit derivation of the moments, variance, and moment generating function of the new model; Section 5 presents the $p$ th quantile function of the new distribution; Section 6 presents the Rényi's entropy of the new distribution; Section 7 presents the $k$ th order statistics of the new distribution; Section 8 proposes the maximum likelihood estimation method for estimating the parameters of the new distribution; Section 9 presents the application of the new distribution to a real data set and a simulation study; Section 10 is the discussion of results and Section 11 contains the conclusion of the study.

\section{Exponentiated Gumbel Type-2 Distribution}

Definition 1. According to Gumbel [7-9], a random variable $X$ is said to follow the Gumbel type-2 distribution if its cumulative density function (cdf) $G(x)$ is given by

$$
G(x)=e^{-\theta x^{-\phi}}, \quad x>0 ; \phi, \theta>0,
$$

while the corresponding probability density function (pdf) $g(x)$ is given by

$$
g(x)=\phi \theta x^{\phi-1} e^{-\theta x^{-\phi}}, \quad x>0 ; \phi, \theta>0 .
$$

Using (3), we obtain the cdf $(F(x))$ of the Exponentiated Gumbel (EG) type-2 distribution as

$$
F(x)=1-\left(1-e^{-\theta x^{-\phi}}\right)^{\alpha}, \quad x>0 ; \alpha, \phi, \theta>0,
$$

while the corresponding pdf $(f(x))$ is given by

$$
\begin{aligned}
& f(x)=\alpha \phi \theta x^{-\phi-1} e^{-\theta x^{-\phi}}\left(1-e^{-\theta x^{-\phi}}\right)^{\alpha-1}, \\
& x>0 ; \alpha, \phi, \theta>0,
\end{aligned}
$$

where $\alpha$ and $\phi$ are the shape parameters and $\theta$ is the scale parameter.

Figure 1 shows the plots of the pdf (a) and cdf (b) of the EG type-2 distribution for certain parameter values.

2.1. Special Cases of the EG Type-2 Distribution. The EG type2 distribution is developed for the purpose of modelling data sets that arise from complex phenomena. It generalizes some standard distributions; for instance, the EG type2 distribution reduces to the Gumbel type- 2 distribution, Exponentiated Fréchet (EF) distribution, and Fréchet distribution when $\alpha=1, \theta=1$, and $\alpha, \theta=1$, respectively.

Theorem 2. If $y=x^{-\phi}$ and $X$ is distributed according to the EG type-2 distribution then, $Y$ is distributed according to the exponentiated exponential (EE) distribution due to Gupta et al. [2].

Proof. The transformation of a random variable $X$ to a random variable $Y$ is defined by $f(y)=f(x) /|d y / d x|$, where $|d y / d x|$ is known as the Jacobian of transformation. Thus, $|d x / d y|=(1 / \phi) y^{-[1 / \phi+1]}$ and

$$
\begin{aligned}
f(y) & =\frac{\alpha \phi \theta y^{1 / \phi+1} e^{-y \theta}\left[1-e^{-y \theta}\right]^{\alpha-1}}{\phi y^{1 / \phi+1}} \\
& =\alpha \theta e^{-y \theta}\left[1-e^{-y \theta}\right]^{\alpha-1} ; \quad y>0, \alpha, \theta>0 .
\end{aligned}
$$

Corollary 3. When $\alpha=1$ (8) reduces to the exponential distribution with parameter $\theta$; that is, $Y \sim \exp (\theta)$.

\section{Some Reliability Properties of the EG Type-2 Distribution}

Reliability theory is generally concerned with the estimation of the probability of longevity or failure of a system.

\subsection{Reliability Function}

Definition 4. The reliability function or the survival function of a random variable $X$ is defined by $R(x)=P(X>x)=$ $1-F(x)$. It could be interpreted as the probability of a system not failing before some specified time $t$, Lee and Wang [10]. The reliability function of the EG type-2 distribution is given by

$$
R(x)=\left(1-e^{-\theta x^{-\phi}}\right)^{\alpha}, \quad x>0 ; \alpha, \phi, \theta>0 .
$$




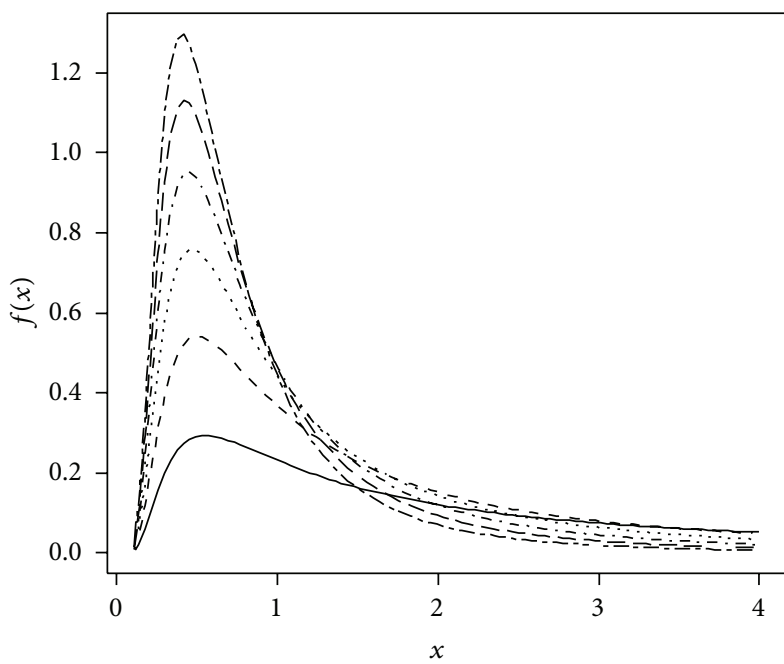

(a)

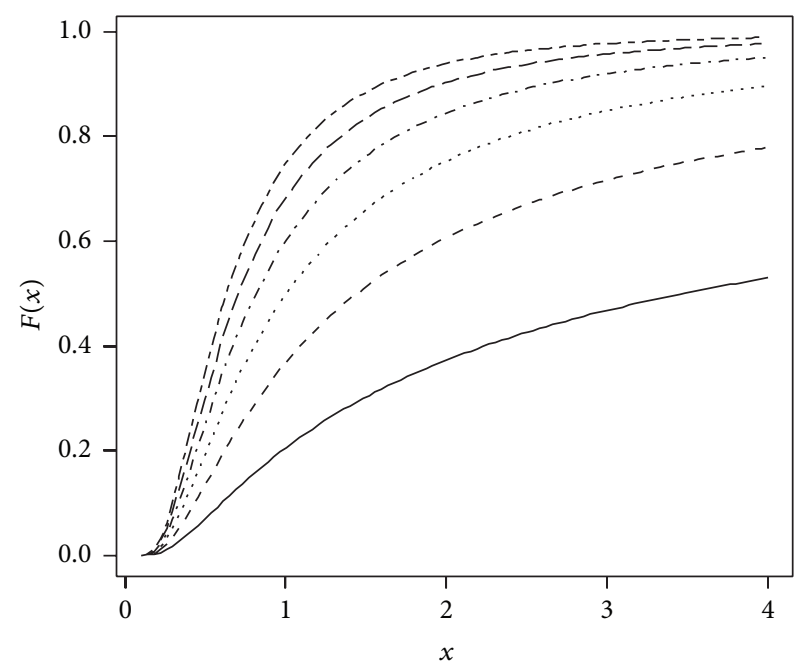

(b)

Figure 1: Possible shapes of the pdf $f(x)$ (a) and $\operatorname{cdf} F(x)$ (b) of the EG type-2 distribution for fixed parameter values of $\phi, \theta=1$ and selected values of $\alpha$ parameter. $\alpha=0.5$ (solid lines), $\alpha=1$ (dashed lines), $\alpha=1.5$ (dotted lines), $\alpha=2$ (dot-dashed lines), $\alpha=2.5$ (long dashed lines), and $\alpha=3$ (two dashed lines).

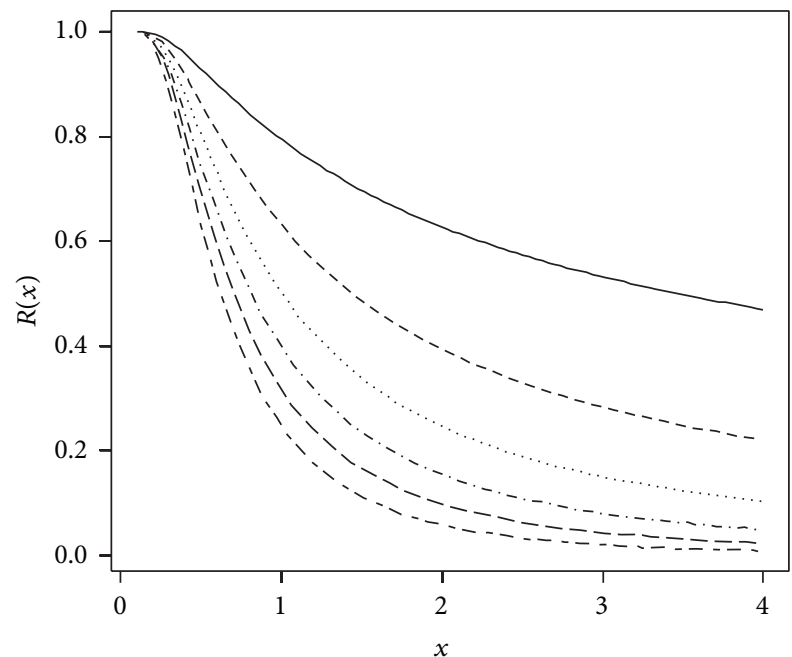

(a)

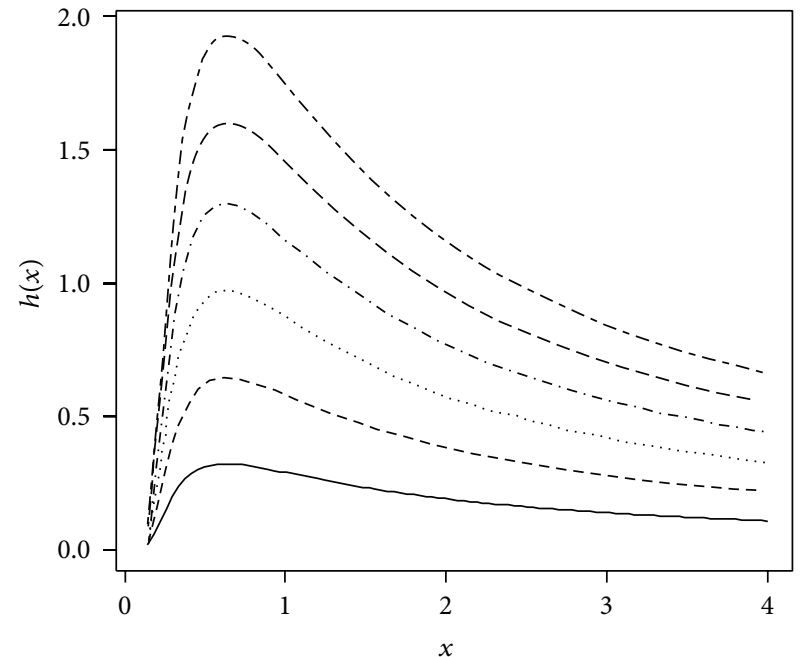

(b)

Figure 2: Possible shapes of the reliability function $R(x)$ (a) and hazard rate function $h(x)$ (b) of the EG type-2 distribution for fixed parameter values of $\phi, \theta=1$ and selected values of $\alpha$ parameter. $\alpha=0.5$ (solid lines), $\alpha=1$ (dashed lines), $\alpha=1.5$ (dotted lines), $\alpha=2$ (dot-dashed lines), $\alpha=2.5$ (long dashed lines), and $\alpha=3$ (two dashed lines).

\subsection{Hazard Rate Function}

Definition 5. The hazard rate function $(h(x))$ or the instantaneous failure rate of a random variable $X$ is the probability that a system fails given that it has survived up to time $t$ and is given by $h(x)=f(x) / R(x)$ (Lee and Wang [10]). Hence, we define the hazard rate function of the EG type-2 distribution as follows:

$$
h(x)=\frac{\alpha \phi \theta x^{-\phi-1} e^{-\theta x^{-\phi}}\left(1-e^{-\theta x^{-\phi}}\right)^{\alpha-1}}{\left(1-e^{-\theta x^{-\phi}}\right)^{\alpha}},
$$

Figure 2 shows plots of the reliability function (a) and hazard rate function (b) of the EG type-2 distribution for selected parameter values.

3.3. Asymptotics. pdf $f(x)$ and cdf $F(x)$ of the EG type2 distribution is unimodal and monotonically increasing, respectively, with increasing values of $\alpha$. The reliability function $R(x)$ of the EG type-2 distribution is 0 as $x \rightarrow 0$ and 1 as $x \rightarrow \infty$. Also, $R(x)$ is a monotonic decreasing function of $x$. For example, when $\alpha=1$,

$$
\begin{aligned}
R(x) & =1-e^{-\theta x^{-\phi}}, \quad x>0 ; \phi, \theta>0 ; \\
\frac{d\left(\left.R(x)\right|_{\alpha=1}\right)}{d x} & =-\phi \theta e^{-\theta x^{-\phi}}<0 .
\end{aligned}
$$

Hence, $R(x)$ is strictly a monotonic decreasing function of $x$. 
The hazard rate function $h(x)$ of the EG type-2 distribution is 0 for both $x \rightarrow 0$ and $x \rightarrow \infty$ and its shape appears increasingly upside-down bathtub with decreasing values of $\alpha$.

\section{The $k$ th Crude Moment of the EG Type-2 Distribution}

In probability theory, the moments of a random variable are one of the most important properties of a distribution that could be used to derive other essential properties such as mean, variance, skewness, and kurtosis statistics that describes a probability distribution. The $k$ th crude moment of a continuous random variable $X$ is defined by $E\left(x^{k}\right)=$ $\int_{-\infty}^{\infty} x^{k} f(x) d x$; then the $k$ th crude moment of the EG type2 distribution follows as

$$
\begin{aligned}
E\left(x^{k}\right) & =\int_{0}^{\infty} x^{k} \alpha \phi \theta x^{-\phi-1} e^{-\theta x^{-\phi}}\left(1-e^{-\theta x^{-\phi}}\right)^{\alpha-1} d x \\
& =\alpha \phi \theta \int_{0}^{\infty} x^{k} x^{-\phi-1} e^{-\theta x^{-\phi}}\left(1-e^{-\theta x^{-\phi}}\right)^{\alpha-1} d x .
\end{aligned}
$$

Substituting $y=\theta x^{-\phi}$ into (13) we have

$$
\begin{aligned}
& E\left(x^{k}\right)=-\frac{\alpha}{\theta^{-k / \phi}} \\
& \cdot \int_{0}^{\infty} y^{-k / \phi} e^{-y}\left(1-e^{-y}\right)^{\alpha}\left(1-e^{-y}\right)^{-1} d y, \\
& E\left(x^{k}\right)=-\frac{\alpha}{\theta^{-k / \phi}} \int_{0}^{\infty} y^{-k / \phi} e^{-y} \sum_{i=0}^{\infty}\left(\begin{array}{c}
\alpha \\
i
\end{array}\right)(-1)^{\alpha-i} e^{y i-\alpha i} \\
& \quad \cdot \sum_{j=0}^{\infty}(-1)^{j}\left(\begin{array}{c}
j+1-1 \\
j
\end{array}\right)(-1)^{-1-j} e^{y+y j} d y
\end{aligned}
$$

and thus,

$$
\begin{aligned}
& E\left(x^{k}\right) \\
& =-\frac{\alpha}{\theta^{-k / \phi}} \sum_{i=0}^{\infty} \sum_{j=0}^{\infty}(-1)^{\alpha-i-1}\left(\begin{array}{c}
\alpha \\
i
\end{array}\right) \int_{0}^{\infty} y^{-k / \phi} e^{-y(\alpha-i-j)} d y .
\end{aligned}
$$

Since $X$ can only take values on the positive real line we can introduce the exponential integral defined by $\operatorname{Ei}(-x)=$ $-\int_{x}^{\infty} t^{-1} e^{-t} d t$ (see Chapter 5 of Abramowitz and Stegun [11] and Equation (6.2.6) of Olver et al. [12]):

$$
\begin{aligned}
& E\left(x^{k}\right) \\
& \quad=\frac{\alpha}{\theta^{-k / \phi}} \sum_{i=0}^{\infty} \sum_{j=0}^{\infty}(-1)^{\alpha-i-1}\left(\begin{array}{c}
\alpha \\
i
\end{array}\right) \int_{x}^{\infty} t^{k / \phi} e^{-t(\alpha-i-j)} d t .
\end{aligned}
$$

Substituting $z=t(\alpha-i-j)$ in (16) we have

$$
\begin{aligned}
E\left(x^{k}\right)=\frac{\alpha}{\theta^{-k / \phi}} \sum_{i=0}^{\infty} \sum_{j=0}^{\infty}(-1)^{\alpha-i-1}\left(\begin{array}{c}
\alpha \\
i
\end{array}\right) \\
\quad \cdot \frac{1}{(\alpha-i-j)^{k / \phi+1}} \int_{x(\alpha-i-j)}^{\infty} z^{k / \phi} e^{-z} d z \\
=\frac{\alpha \Gamma(\alpha+1)}{\theta^{-k / \phi}} \\
\quad \cdot \sum_{i=0}^{\infty} \sum_{j=0}^{\infty} \frac{(-1)^{\alpha-i-1} \Gamma(k / \phi+1 ; x(\alpha-i-j))}{\Gamma(i+1) \Gamma(\alpha-i+1)(\alpha-i-j)^{k / \phi+1}} .
\end{aligned}
$$

Thus, evaluating (18) at $k=1$ and $k=2$ yields the mean $E(x)$ and second crude moment $E\left(x^{2}\right)$ then we can obtain the variance $V(x)$ of the EG type-2 distribution as $V(x)=E\left(x^{2}\right)-$ $(E(x))^{2}$. Denoting $E\left(x^{k}\right)$ by $\mu_{k}^{\prime}$ the coefficient of variation (cv), skewness $\left(\gamma_{1}\right)$, and kurtosis $\left(\gamma_{2}\right)$ statistics of the EG type-2 distribution can be obtained by evaluating

$$
\begin{aligned}
& \mathrm{cv}=\sqrt{\frac{\mu_{2}^{\prime}}{\mu_{1}^{\prime 2}}-1,} \\
& \gamma_{1}=\frac{\mu_{3}^{\prime}-3 \mu_{2}^{\prime} \mu_{1}^{\prime}+2 \mu_{1}^{\prime 3}}{\left(\mu_{2}^{\prime}-\mu_{1}^{\prime 2}\right)^{3 / 2}}, \\
& \gamma_{2}=\frac{\mu_{4}^{\prime}-4 \mu_{3}^{\prime} \mu_{1}^{\prime}+6 \mu_{2}^{\prime} \mu_{1}^{\prime 2}-3 \mu_{1}^{\prime 4}}{\left(\mu_{2}^{\prime}-\mu_{1}^{\prime 2}\right)^{2}},
\end{aligned}
$$

respectively.

4.1. The Moment Generating Function of the EG Type-2 Distribution. Generally, the moment generating function (mgf) denoted by $M_{x}(t)$ of a random variable $X$ is defined as

$$
M_{x}(t)=E\left(e^{t x}\right)=E\left(\sum_{k=0}^{\infty} \frac{(t x)^{k}}{k !}\right)
$$

$$
=\sum_{k=0}^{\infty} \frac{t^{k}}{k !} E\left(x^{k}\right) .
$$

If a random variable $X$ is distributed according to the EG Type- 2 distribution, then its mgf is given by

$$
\begin{aligned}
& M_{x}(t)=\alpha \Gamma(\alpha+1) \\
& \cdot \sum_{i=0}^{\infty} \sum_{j=0}^{\infty} \sum_{k=0}^{\infty} \frac{(-1)^{\alpha-i-1} t^{k} \Gamma(k / \phi+1 ; x(\alpha-i-j))}{\Gamma(k+1) \Gamma(i+1) \Gamma(\alpha-i+1)(\alpha-i-j)^{k / \phi+1} \theta^{-k / \phi}} .
\end{aligned}
$$

\section{The $p$ th Quantile Function of the EG Type-2 Distribution}

The $p$ th quantile function $x_{p}$ of the EG Type- 2 distribution is the inverse of (5) and it is obtained as

$$
x_{p}=\left(-\frac{1}{\theta} \ln \left(1-(1-p)^{1 / \alpha}\right)\right)^{-1 / \phi} .
$$


We can simulate random variables from the EG type-2 distribution through the inversion of the cdf method by simply replacing $p$ in $(24)$ with $U(0,1)$ variates. Also, evaluating $(24)$ at $p=1 / 2$ gives the median of the distribution.

\section{The Rényi Entropy}

The Rényi entropy is used to measure uncertainty or variation in a random variable $X$. The Rényi's entropy measure has been shown to be effective in comparing the tails and shapes of various standard distributions, Song [13]. The Rényi entropy measure for a continuous random variable $X$ is given by

$$
\begin{aligned}
H_{\lambda}(x) & =\lim _{n \rightarrow \infty}\left(I_{\lambda}\left(f_{n}\right)-\ln (n)\right) \\
& =\frac{1}{1-\lambda} \ln \int f^{\lambda}(x) d x .
\end{aligned}
$$

Then the Rényi entropy measure for the EG type-2 distribution could be obtained as follows:

$$
\begin{aligned}
& H_{\lambda}(x)=\frac{1}{1-\lambda} \\
& \quad \cdot \ln \int_{0}^{\infty}\left(\alpha \phi \theta x^{-\phi-1} e^{-\theta x^{-\phi}}\left(1-e^{-\theta x^{-\phi}}\right)^{\alpha-1}\right)^{\lambda} d x .
\end{aligned}
$$

Setting $I_{\lambda}=\int_{0}^{\infty}\left(\alpha \phi \theta x^{-\phi-1} e^{-\theta x^{-\phi}}\left(1-e^{-\theta x^{-\phi}}\right)^{\alpha-1}\right)^{\lambda} d x$ in (26) we have

$$
I_{\lambda}=(\alpha \phi \theta)^{\lambda} \int_{0}^{\infty} x^{-\lambda \phi-\lambda} e^{-\lambda \theta x^{-\phi}}\left(1-e^{-\theta x^{-\phi}}\right)^{\lambda \alpha-\lambda} d x .
$$

Substituting $y=\theta x^{-\phi}$ in (27) we have

$$
\begin{aligned}
I_{\lambda} & =-\frac{(\alpha \phi \theta)^{\lambda}}{\phi \theta^{\lambda / \phi-1 / \phi+\lambda-1}} \\
& \cdot \int_{0}^{\infty} y^{\lambda / \phi-1 / \phi+\lambda-1} e^{-\lambda y}\left(1-e^{-y}\right)^{\lambda \alpha}\left(1-e^{-y}\right)^{-\lambda} d y, \\
I_{\lambda} & =-\frac{(\alpha \phi \theta)^{\lambda}}{\phi \theta^{\lambda / \phi-1 / \phi+\lambda-1}} \\
& \cdot \int_{0}^{\infty} y^{\lambda / \phi-1 / \phi+\lambda-1} e^{-y} \sum_{i=0}^{\infty}\left(\begin{array}{c}
\lambda \alpha \\
i
\end{array}\right)(-1)^{\lambda \alpha-i} e^{-y \lambda \alpha+y i} \\
& \cdot \sum_{j=0}^{\infty}(-1)^{j}\left(\begin{array}{c}
j+\lambda-1 \\
j
\end{array}\right)(-1)^{-\lambda-j} e^{\lambda y+j y} d y, \\
I_{\lambda} & =-\frac{(\alpha \phi \theta)^{\lambda}}{\phi \theta^{\lambda / \phi-1 / \phi+\lambda-1}} \\
& \cdot \sum_{i=0}^{\infty} \sum_{j=0}^{\infty}(-1)^{\lambda \alpha-\lambda-i}\left(\begin{array}{c}
\lambda \alpha \\
i
\end{array}\right)\left(\begin{array}{c}
j+\lambda-1 \\
j
\end{array}\right) \\
& \cdot \int_{0}^{\infty} y^{\lambda / \phi-1 / \phi+\lambda-1} e^{-y(\lambda \alpha-i-j)} d y
\end{aligned}
$$

using the expression for the $E i(-x)$ function defined in Section 4; we have

$$
\begin{aligned}
I_{\lambda}= & \frac{(\alpha \phi \theta)^{\lambda}}{\phi \theta^{\lambda / \phi-1 / \phi+\lambda-1}} \\
& \cdot \sum_{i=0}^{\infty} \sum_{j=0}^{\infty}(-1)^{\lambda \alpha-\lambda-i}\left(\begin{array}{c}
\lambda \alpha \\
i
\end{array}\right)\left(\begin{array}{c}
j+\lambda-1 \\
j
\end{array}\right) \\
& \cdot \int_{x}^{\infty} t^{\lambda / \phi-1 / \phi+\lambda-1} e^{-t(\lambda \alpha-i-j)} d t
\end{aligned}
$$

and substituting $z=t(\lambda \alpha-i-j)$ in (29) we have

$$
\begin{aligned}
I_{\lambda}= & \frac{(\alpha \phi \theta)^{\lambda}}{\phi \theta^{\lambda / \phi-1 / \phi+\lambda-1}} \\
& \cdot \sum_{i=0}^{\infty} \sum_{j=0}^{\infty}(-1)^{\lambda \alpha-\lambda-i}\left(\begin{array}{c}
\lambda \alpha \\
i
\end{array}\right)\left(\begin{array}{c}
j+\lambda-1 \\
j
\end{array}\right) \\
& \cdot \frac{1}{(\lambda \alpha-i-j)^{1 / \phi-\lambda / \phi-\lambda+2}} \\
& \cdot \int_{x(\lambda \alpha-i-j)}^{\infty} z^{1 / \phi-\lambda / \phi-\lambda+1} e^{-z} d z, \\
I_{\lambda}= & \frac{(\alpha \phi \theta)^{\lambda}}{\phi \theta^{\lambda / \phi-1 / \phi+\lambda-1}} \\
& \cdot \sum_{i=0}^{\infty} \sum_{j=0}^{\infty}(-1)^{\lambda \alpha-\lambda-i}\left(\begin{array}{c}
\lambda \alpha \\
i
\end{array}\right)\left(\begin{array}{c}
j+\lambda-1 \\
j
\end{array}\right) \\
& \cdot \frac{\Gamma\left(\frac{1}{\phi}-\frac{\lambda}{\phi}-\lambda+2 ; x(\lambda \alpha-i-j)\right)}{(\lambda \alpha-i-j)^{1 / \phi-\lambda / \phi-\lambda+2}} \\
& \frac{1}{i}{ }^{\lambda}
\end{aligned}
$$

and thus,

$$
\begin{aligned}
& H_{\lambda}(x)=\frac{1}{1-\lambda} \ln \left(\frac{(\alpha \phi \theta)^{\lambda}}{\phi \theta^{\lambda / \phi-1 / \phi+\lambda-1}}\right. \\
& \left.\cdot \sum_{i=0}^{\infty} \sum_{j=0}^{\infty}(-1)^{\lambda \alpha-\lambda-i}\left(\begin{array}{c}
\lambda \alpha \\
i
\end{array}\right)\left(\begin{array}{c}
j+\lambda-1 \\
j
\end{array}\right)\right)+\frac{1}{1-\lambda} \\
& \cdot \ln \left(\frac{1}{(\lambda \alpha-i-j)^{1 / \phi-\lambda / \phi-\lambda+2}}\right. \\
& \left.\cdot \Gamma\left(\frac{1}{\phi}-\frac{\lambda}{\phi}-\lambda+2 ; x(\lambda \alpha-i-j)\right)\right) .
\end{aligned}
$$




\section{The Order Statistics of the EG Type-2 Distribution}

The distribution of the $k$ th order statistics $f_{X(k)}(x)$ of a random sample $X_{1}, X_{2}, \ldots, X_{n}$ of size $n$ is generally given as

$$
\begin{aligned}
& f_{X(k)}(x) \\
& =\frac{n !}{(k-1) !(n-k) !} f_{x}(x)\left(F_{x}(x)\right)^{k-1}\left(1-F_{x}(x)\right)^{n-k} .
\end{aligned}
$$

Hence, the density of the $k$ th order statistics of the EG Type-2 distribution is given by

$$
\begin{aligned}
& f_{X(k)}(x)=\frac{n !}{(k-1) !(n-k) !} \\
& \cdot \alpha \phi \theta x^{-\phi-1} e^{-\theta x^{-\phi}}\left(1-\left(1-e^{-\theta x^{-\phi}}\right)^{\alpha}\right)^{k-1} \\
& \cdot\left(1-e^{-\theta x^{-\phi}}\right)^{\alpha(n-k+1)-1}, \quad x>0 ; \alpha, \phi, \theta>0 .
\end{aligned}
$$

The density of the $k$ th smallest order statistics of the EG Type2 distribution is given by

$$
\begin{array}{r}
f_{X(1)}(x)=n \alpha \phi \theta x^{-\phi-1} e^{-\theta x^{-\phi}}\left(1-e^{-\theta x^{-\phi}}\right)^{\alpha n-1}, \\
x>0 ; \alpha, \phi, \theta>0 .
\end{array}
$$

The density of the $k$ th largest order statistics of the EG Type-2 distribution is given by

$$
\begin{aligned}
f_{X(n)}(x)= & n \alpha \phi \theta x^{-\phi-1} e^{-\theta x^{-\phi}}\left(1-e^{-\theta x^{-\phi}}\right)^{\alpha-1} \\
& \cdot\left(1-\left(1-e^{-\theta x^{-\phi}}\right)^{\alpha}\right)^{n-1},
\end{aligned}
$$

$$
x>0 ; \alpha, \phi, \theta>0 .
$$

\section{Parameter Estimation of the EG Type-2 Distribution}

In this section, we propose the method of maximum likelihood estimates (MLE) for the estimation of the parameters of the EG type-2 distribution. Suppose a random variable $X$ of size $n$ has the EG type-2 distribution then, its MLE are obtained as follows:

The likelihood function is given by

$$
\begin{aligned}
& L(x ; \alpha, \phi, \theta)=\prod_{i=1}^{n} \alpha \phi \theta x_{i}^{-\phi-1} e^{-\theta x_{i}^{-\phi}}\left(1-e^{-\theta x_{i}^{-\phi}}\right)^{\alpha-1} \\
& \quad=(\alpha \phi \theta)^{n} e^{-\theta \sum_{i=1}^{n} x_{i}^{-\phi}} \prod_{i=1}^{n} x_{i}^{-\phi-1}\left(1-e^{-\theta x_{i}^{-\phi}}\right)^{\alpha-1}
\end{aligned}
$$

with the corresponding log-likelihood function

$$
\begin{aligned}
\ln L(x ; \alpha, \phi, \theta)= & n \ln (\alpha \phi \theta)-\theta \sum_{i=1}^{n} x_{i}^{-\phi} \\
& -(\phi+1) \sum_{i=1}^{n} \ln \left(x_{i}\right) \\
& +\sum_{i=1}^{n} \ln \left(1-e^{-\theta x_{i}^{-\phi}}\right)^{\alpha-1} .
\end{aligned}
$$

Taking the partial derivatives of the log-likelihood function with respect to $\alpha, \phi$, and $\theta$, respectively, and equating to 0 give

$$
\begin{aligned}
& \frac{\partial \ln (L(x ; \alpha, \phi, \theta))}{\partial \alpha} \\
& =\frac{n}{\alpha}+\sum_{i=1}^{n} \frac{\left(1-e^{-\theta x_{i}^{-\phi}}\right)^{\alpha-1} \ln \left(1-e^{-\theta x_{i}^{-\phi}}\right)}{\left(1-\mathrm{e}^{-\theta x_{i}^{-\phi}}\right)^{\alpha-1}}=0, \\
& \frac{\partial \ln (L(x ; \alpha, \phi, \theta))}{\partial \phi} \\
& =\frac{n}{\phi}+\theta \sum_{i=1}^{n} x_{i}^{-\phi} \ln \left(x_{i}\right)-\sum_{i=1}^{n} \ln \left(x_{i}\right) \\
& \quad-\sum_{i=1}^{n} \frac{(\alpha-1) \theta x_{i}^{-\phi} \ln \left(x_{i}\right) e^{-\theta x_{i}^{-\phi}}\left(1-e^{-\theta x_{i}^{-\phi}}\right)^{\alpha-2}}{\left(1-e^{-\theta x_{i}^{-\phi}}\right)^{\alpha-1}} \\
& =0,
\end{aligned}
$$

$$
\begin{aligned}
& \frac{\partial \ln (L(x ; \alpha, \phi, \theta))}{\partial \theta} \\
& =\frac{n}{\theta}-\sum_{i=1}^{n} x_{i}^{-\phi} \\
& +\sum_{i=1}^{n} \frac{(\alpha-1) x_{i}^{-\phi} e^{-\theta x_{i}^{-\phi}}\left(1-e^{-\theta x_{i}^{-\phi}}\right)^{\alpha-2}}{\left(1-e^{-\theta x_{i}^{-\phi}}\right)^{\alpha-1}}=0 .
\end{aligned}
$$

Equations (38) can only be solved by some numerical optimization methods such as Newton Raphson's algorithm to obtain the MLE of $\alpha, \phi$, and $\theta$. 
TABLE 1: Survival times in months of 20 acute myeloid leukemia patients.

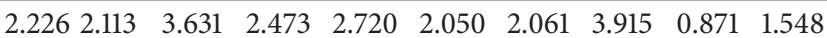

$\begin{array}{llllllllll}2.746 & 1.972 & 2.265 & 1.200 & 2.967 & 2.808 & 1.079 & 2.353 & 0.726 & 1.958\end{array}$

\section{Application}

In this section we would fit the EG type-2 distribution to a real and uncensored data set to demonstrate its applicability and flexibility. The goodness of fit of the new distribution would be compared with the three submodels, namely, the Gumbel type-2 distribution, Exponentiated Fréchet distribution, and Fréchet distribution and two other related heavy tail distributions: Weibull distribution $f(x)=$ $\alpha / \beta(x / \beta)^{\alpha-1} \exp \left(-(x / \beta)^{\alpha}\right), x>0, \alpha>0$, and $\beta>0$, and lognormal (LN) distribution $f(x)=1 /(x \sigma \sqrt{2 \pi}) \exp (-[\ln (x)-$ $\left.\mu] / 2 \sigma^{2}\right), x>0, \mu \in \Re$, and $\sigma>0$. The model comparison would be based on the minimized log-likelihood estimate and the following information statistics: AIC by Akaike [14], AICC by Sugiura [15], CAIC by Bozdogan [16], HQC by Schwarz [17], and BIC by Hannan and Quinn [18]. The model with the smallest minimized loglikelihood and information statistics value is the best. The data set in Table 1 shows the survival times in months of 20 acute myeloid leukemia patients reported in Afify et al. [19].

9.1. Monte-Carlo Simulation. In this section we present a Monte-Carlo simulation study to investigate the effect of sample size on the maximum likelihood estimates of the parameters of the EG type-2 distribution and further to assess the stability of these parameters. Different sample sizes $(25,50,75,100, \ldots, 500)$ were drawn from the EG type-2 distribution with parameters $\alpha=1.50, \theta=$ 1.50 , and $\phi=1.50$ using the inverse transformation method with (24) where each sample was replicated 5000 times. Using the simulated random variables we estimate the parameters of the EG type-2 distribution through the method of maximum likelihood estimation and the procedure was repeated 5000 times for each sample size. The mean (parameter estimate) and standard deviation (standard error (se)) of the 5000 parameters each for $\alpha, \theta$, and $\phi$ for each sample size were computed and the result is presented in Table 3. Furthermore, the corresponding bias and mean square errors (mse) of each of the parameter estimates are tabulated in Table 4. Analogously, Tables 5 and 6 show simulation results for the EG type-2 distribution with parameters $\alpha=3.00, \theta=4.00$, and $\phi=$ 5.00 .

\section{Discussion of Results}

From the pdf and cdf plots in Figure 1, the pdf of the EG type-2 distribution is unimodal and increasingly unimodal for increasing values of $\alpha$ (shape parameter) while its cdf is monotonic increasing and more monotonically

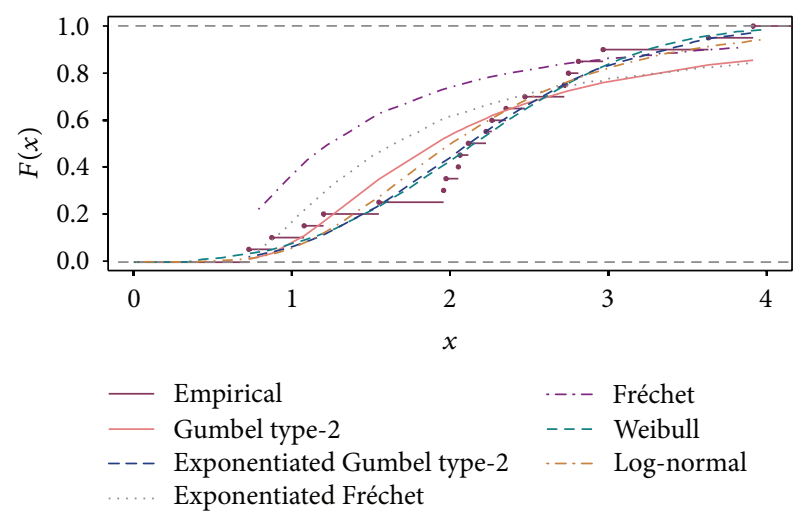

FIGURE 3: cdf plots of the fitted distributions superimposed on the empirical density plot of the survival times data.

increasing for increasing values of $\alpha$. Also, the plots of the reliability function $(R(x))$ and hazard rate function $(h(x))$ in Figure 2 show that $R(x)$ is monotonic decreasing and more monotonically decreasing for increasing values of $\alpha$ while $h(x)$ is upside-down bathtub and becomes more upside-down bathtub for decreasing values of $\alpha$. Results from the model fittings as tabulated in Table 2 indicate that the EG type-2 distribution provides the best fit to the data based on its smallest minimized log-likelihood and information statistics values. Figure 3 depicts the cdf's of all the estimated distributions in Table 2 superimposed on the empirical cdf of the data, where the cdf of the EG type-2 distribution is closely aligned to the empirical one than the other distributions. From the simulation results in Tables 3, 4, 5, and 6 it is clear that the parameters of the EG type-2 distribution approach the true value as the sample size increases, while the standard error, bias, and the mse decrease down the column with increasing sample size.

\section{Conclusion}

This paper introduces a new lifetime distribution, the Exponentiated Gumbel (EG) type-2 distribution. The new distribution generalizes the standard Gumbel type- 2 distribution and has the following distributions as special cases: Gumbel type-2 distribution, Exponentiated Fréchet distribution, and Fréchet distribution. We have provided explicit mathematical expressions for some of its basic statistical properties such as the probability density function, cumulative density function, $k$ th crude moment, variance, coefficient of variation, skewness, kurtosis, moment generating function, and $p$ th quantile function and some reliability characteristics like the reliability and hazard rate functions. Estimation of the model parameters was approached through the method of maximum likelihood estimates. The flexibility and applicability of the new lifetime distribution were illustrated with a real data set and the results obtained revealed that the EG type-2 distribution provides the best fit among all the compared related distributions. We recommend the EG type-2 distribution for modelling complex data sets and 
TABLE 2: Results from the survival times data fitting.

\begin{tabular}{|c|c|c|c|c|c|c|c|}
\hline Models & Estimates & $-\widehat{\ell}$ & AIC & AICC & CAIC & HQC & $\mathrm{BIC}$ \\
\hline \multicolumn{8}{|c|}{ EG type-2 } \\
\hline$\widehat{\alpha}$ & $2.0263 \times 10^{4}$ & & & & & & \\
\hline$\hat{\theta}$ & 12.7096 & 24.12718 & 52.25436 & 52.96024 & 56.24583 & 52.64312 & 54.24583 \\
\hline$\widehat{\phi}$ & 0.2821 & & & & & & \\
\hline \multicolumn{8}{|l|}{ G type-2 } \\
\hline$\hat{\theta}$ & 2.6040 & 29.08667 & 62.17334 & 62.87922 & 66.16481 & 62.5621 & 64.16481 \\
\hline$\widehat{\phi}$ & 2.0651 & & & & & & \\
\hline \multicolumn{8}{|l|}{$E F$} \\
\hline$\widehat{\alpha}$ & 0.3928 & 31.89448 & 67.78896 & 68.49484 & 71.78042 & 68.17771 & 69.78042 \\
\hline$\widehat{\phi}$ & 3.4393 & & & & & & \\
\hline \multicolumn{8}{|l|}{ Fréchet } \\
\hline$\widehat{\phi}$ & 1.7378 & 35.45610 & 72.91219 & 73.13442 & 74.90793 & 73.10657 & 76.90366 \\
\hline \multicolumn{8}{|l|}{$L N$} \\
\hline$\widehat{\mu}$ & 0.6971 & 25.71549 & 55.43098 & 56.13687 & 59.42245 & 55.81974 & 57.42245 \\
\hline$\widehat{\sigma}$ & 0.4360 & & & & & & \\
\hline \multicolumn{8}{|l|}{ Weibull } \\
\hline$\widehat{\alpha}$ & 2.9237 & 24.30433 & 54.60867 & 56.10867 & 60.59587 & 55.1918 & 54.60013 \\
\hline$\widehat{\beta}$ & 2.4502 & & & & & & \\
\hline
\end{tabular}

TABLE 3: Monte-Carlo simulation results of the parameter estimates and standard errors of the EG type-2 distribution with parameters: $\alpha=1.50, \theta=1.50$, and $\phi=1.50$ for different sample sizes.

\begin{tabular}{lcccccc}
\hline Sample size & $\widehat{\alpha}$ & $\widehat{\theta}$ & $\widehat{\phi}$ & $\mathrm{se}_{\widehat{\alpha}}$ & $\mathrm{se}_{\widehat{\theta}}$ & $\mathrm{se}_{\hat{\phi}}$ \\
\hline 25 & 292.3201 & 1.9186 & 2.5361 & 2608.3737 & 1.8417 & 2.7992 \\
50 & 16.7755 & 1.6267 & 1.8183 & 600.1862 & 0.9444 & 1.1577 \\
75 & 2.1483 & 1.5741 & 1.6634 & 5.8997 & 0.6814 & 0.6847 \\
100 & 1.8196 & 1.5463 & 1.6125 & 1.5079 & 0.5516 & 0.5114 \\
125 & 1.7586 & 1.5401 & 1.5843 & 1.2131 & 0.4976 & 0.4302 \\
150 & 1.6970 & 1.5334 & 1.5684 & 0.9432 & 0.4464 & 0.3834 \\
175 & 1.6660 & 1.5332 & 1.5538 & 0.7793 & 0.4072 & 0.3466 \\
200 & 1.6269 & 1.5208 & 1.5506 & 0.6975 & 0.3728 & 0.3154 \\
225 & 1.6252 & 1.5272 & 1.5386 & 0.6501 & 0.3536 & 0.2952 \\
250 & 1.6102 & 1.5251 & 1.5331 & 0.5715 & 0.3268 & 0.2728 \\
275 & 1.5909 & 1.5168 & 1.5344 & 0.5333 & 0.3119 & 0.2597 \\
300 & 1.5825 & 1.5152 & 1.5320 & 0.5108 & 0.3025 & 0.2511 \\
325 & 1.5755 & 1.5152 & 1.5273 & 0.4798 & 0.2870 & 0.2363 \\
350 & 1.5730 & 1.5146 & 1.5271 & 0.4624 & 0.2791 & 0.2294 \\
375 & 1.5635 & 1.5104 & 1.5255 & 0.4404 & 0.2687 & 0.2205 \\
400 & 1.5603 & 1.5129 & 1.5221 & 0.4180 & 0.2558 & 0.2094 \\
425 & 1.5517 & 1.5086 & 1.5237 & 0.4034 & 0.2491 & 0.2031 \\
450 & 1.5498 & 1.5096 & 1.5213 & 0.3873 & 0.2405 & 0.1966 \\
475 & 1.5450 & 1.5079 & 1.5220 & 0.3781 & 0.2361 & 0.1927 \\
500 & 1.5433 & 1.5069 & 1.5191 & 0.3620 & 0.2272 & 0.1862 \\
\hline & & & & & &
\end{tabular}

TABLE 4: Monte-Carlo simulation results of the estimators bias and mse of the EG type-2 distribution with parameters: $\alpha=1.50, \theta=$ 1.50 , and $\phi=1.50$ for different sample sizes.

\begin{tabular}{lcccccc}
\hline $\begin{array}{l}\text { Sample } \\
\text { size }\end{array}$ & bias $_{\widehat{\alpha}}$ & bias $_{\hat{\theta}}$ & bias $_{\hat{\phi}}$ & mse $_{\widehat{\alpha}}$ & mse $_{\widehat{\theta}}$ & mse $_{\hat{\phi}}$ \\
\hline 25 & 290.8201 & 0.4186 & 1.0361 & 6887509.5404 & 3.5666 & 8.9085 \\
50 & 15.2755 & 0.1267 & 0.3183 & 360420.7341 & 0.9078 & 1.4415 \\
75 & 0.6483 & 0.0741 & 0.1634 & 35.2238 & 0.4698 & 0.4955 \\
100 & 0.3196 & 0.0463 & 0.1125 & 2.3757 & 0.3064 & 0.2742 \\
125 & 0.2586 & 0.0401 & 0.0843 & 1.5383 & 0.2492 & 0.1922 \\
150 & 0.1970 & 0.0334 & 0.0684 & 0.9283 & 0.2004 & 0.1516 \\
175 & 0.1660 & 0.0332 & 0.0538 & 0.6348 & 0.1669 & 0.1230 \\
200 & 0.1269 & 0.0208 & 0.0506 & 0.5025 & 0.1394 & 0.1020 \\
225 & 0.1252 & 0.0272 & 0.0386 & 0.4383 & 0.1257 & 0.0886 \\
250 & 0.1102 & 0.0251 & 0.0331 & 0.3388 & 0.1074 & 0.0755 \\
275 & 0.0909 & 0.0168 & 0.0344 & 0.2927 & 0.0976 & 0.0686 \\
300 & 0.0825 & 0.0152 & 0.0320 & 0.2677 & 0.0917 & 0.0641 \\
325 & 0.0755 & 0.0152 & 0.0273 & 0.2359 & 0.0826 & 0.0566 \\
350 & 0.0730 & 0.0146 & 0.0271 & 0.2191 & 0.0781 & 0.0534 \\
375 & 0.0635 & 0.0104 & 0.0255 & 0.1980 & 0.0723 & 0.0493 \\
400 & 0.0603 & 0.0129 & 0.0221 & 0.1784 & 0.0656 & 0.0443 \\
425 & 0.0517 & 0.0086 & 0.0237 & 0.1654 & 0.0621 & 0.0418 \\
450 & 0.0498 & 0.0096 & 0.0213 & 0.1524 & 0.0579 & 0.0391 \\
500 & 0.0450 & 0.0079 & 0.0220 & 0.1449 & 0.0558 & 0.0376 \\
\hline 55 & 0.0433 & 0.0069 & 0.0191 & 0.1329 & 0.0517 & 0.0350 \\
\hline
\end{tabular}


TABLE 5: Monte-Carlo simulation results of the parameter estimates and standard errors of the EG type-2 distribution with parameters: $\alpha=3.00, \theta=4.00$, and $\phi=5.00$ for different sample sizes.

\begin{tabular}{lcccccc}
\hline $\begin{array}{l}\text { Sample } \\
\text { size }\end{array}$ & $\hat{\alpha}$ & $\hat{\theta}$ & $\hat{\phi}$ & $\mathrm{se}_{\hat{\alpha}}$ & $\mathrm{se}_{\hat{\theta}}$ & $\mathrm{se}_{\hat{\phi}}$ \\
\hline 25 & 957.2346 & 9.1225 & 8.1140 & 4764.4832 & 171.3331 & 10.5485 \\
50 & 118.7143 & 4.4324 & 5.8491 & 1677.2419 & 1.5587 & 3.6720 \\
75 & 23.7643 & 4.2555 & 5.3847 & 804.2310 & 0.8669 & 2.1538 \\
100 & 4.9487 & 4.1628 & 5.2897 & 27.8859 & 0.6844 & 1.6550 \\
125 & 4.3378 & 4.1331 & 5.2224 & 16.5547 & 0.5964 & 1.4261 \\
150 & 3.7404 & 4.1022 & 5.1856 & 3.4572 & 0.5251 & 1.2608 \\
175 & 3.6405 & 4.0926 & 5.1459 & 3.0479 & 0.4868 & 1.1427 \\
200 & 3.5112 & 4.0785 & 5.1310 & 2.1757 & 0.4479 & 1.0647 \\
225 & 3.4261 & 4.0684 & 5.1207 & 2.0217 & 0.4161 & 0.9853 \\
250 & 3.3987 & 4.0598 & 5.0902 & 1.6941 & 0.3987 & 0.9206 \\
275 & 3.3271 & 4.0538 & 5.0939 & 1.5224 & 0.3699 & 0.8769 \\
300 & 3.2975 & 4.0468 & 5.0901 & 1.4489 & 0.3588 & 0.8396 \\
325 & 3.2727 & 4.0499 & 5.0834 & 1.3216 & 0.3432 & 0.8059 \\
350 & 3.2506 & 4.0458 & 5.0683 & 1.2191 & 0.3264 & 0.7609 \\
375 & 3.2390 & 4.0398 & 5.0645 & 1.1950 & 0.3221 & 0.7385 \\
400 & 3.2020 & 4.0337 & 5.0716 & 1.1148 & 0.3050 & 0.7162 \\
425 & 3.2035 & 4.0379 & 5.0564 & 1.0529 & 0.2926 & 0.6887 \\
450 & 3.2039 & 4.0344 & 5.0396 & 1.0356 & 0.2880 & 0.6670 \\
475 & 3.1827 & 4.0328 & 5.0502 & 0.9930 & 0.2797 & 0.6472 \\
500 & 3.1648 & 4.0334 & 5.0533 & 0.9531 & 0.2724 & 0.6326 \\
\hline & & & & & &
\end{tabular}

TABLE 6: Monte-Carlo simulation results of the estimators bias and mse of the EG type-2 distribution with parameters: $\alpha=3.00, \theta=$ 4.00 , and $\phi=5.00$ for different sample sizes.

\begin{tabular}{lcccccc}
\hline $\begin{array}{l}\text { Sample } \\
\text { size }\end{array}$ & bias $_{\hat{\alpha}}$ & bias $_{\hat{\theta}}$ & bias $_{\hat{\phi}}$ & mse $_{\hat{\alpha}}$ & mse $_{\hat{\theta}}$ & mse $_{\hat{\phi}}$ \\
\hline 25 & 954.2346 & 5.1225 & 3.1140 & $\begin{array}{c}2.360859 \times \\
10^{7}\end{array}$ & 29378.3413 & 120.9573 \\
& & & & & \\
50 & 115.7143 & 0.4324 & 0.8491 & $\begin{array}{c}2.826249 \times \\
10^{6}\end{array}$ & 2.6162 & 14.2029 \\
75 & 20.7643 & 0.2555 & 0.3847 & $\begin{array}{c}6.71540 \times \\
10^{5}\end{array}$ & 0.8168 & 4.7864 \\
100 & 1.9487 & 0.1628 & 0.2897 & 781.3449 & 0.4949 & 2.8228 \\
125 & 1.3378 & 0.1331 & 0.2224 & 275.8201 & 0.3733 & 2.0829 \\
150 & 0.7404 & 0.1022 & 0.1856 & 12.4991 & 0.2861 & 1.6240 \\
175 & 0.6405 & 0.0926 & 0.1459 & 9.6992 & 0.2455 & 1.3270 \\
200 & 0.5112 & 0.0785 & 0.1310 & 4.9943 & 0.2067 & 1.1506 \\
225 & 0.4261 & 0.0684 & 0.1207 & 4.2683 & 0.1778 & 0.9854 \\
250 & 0.3987 & 0.0598 & 0.0902 & 3.0285 & 0.1626 & 0.8556 \\
275 & 0.3271 & 0.0538 & 0.0939 & 2.4246 & 0.1397 & 0.7778 \\
300 & 0.2975 & 0.0468 & 0.0901 & 2.1878 & 0.1309 & 0.7130 \\
325 & 0.2727 & 0.0499 & 0.0834 & 1.8209 & 0.1202 & 0.6564 \\
350 & 0.2506 & 0.0458 & 0.0683 & 1.5489 & 0.1086 & 0.5836 \\
375 & 0.2390 & 0.0398 & 0.0645 & 1.4850 & 0.1053 & 0.5496 \\
400 & 0.2020 & 0.0337 & 0.0716 & 1.2834 & 0.0941 & 0.5181 \\
425 & 0.2035 & 0.0379 & 0.0564 & 1.1500 & 0.0870 & 0.4774 \\
450 & 0.2039 & 0.0344 & 0.0396 & 1.1138 & 0.0841 & 0.4465 \\
475 & 0.1827 & 0.0328 & 0.0502 & 1.0193 & 0.0793 & 0.4213 \\
500 & 0.1648 & 0.0334 & 0.0533 & 0.9355 & 0.0753 & 0.4029 \\
\hline
\end{tabular}

hope that it would receive significant applications in the future.

\section{Competing Interests}

The authors declare that there are no competing interests regarding the publication of this paper.

\section{References}

[1] E. C. Pinheiro and S. L. P. Ferrari, "A comparative review of generalizations of the Gumbel extreme value distribution with an application to wind speed data," Journal of Statistical Computation and Simulation, 2015.

[2] R. C. Gupta, P. L. Gupta, and R. D. Gupta, "Modeling failure time data by Lehman alternatives," Communications in Statistics-Theory and Methods, vol. 27, no. 4, pp. 887-904, 1998.

[3] S. Nadarajah and S. Kotz, "The exponentiated type distributions," Acta Applicandae Mathematica, vol. 92, no. 2, pp. 97-111, 2006.

[4] S. Nadarajah, "The exponentiated Gumbel distribution with climate application," Environmetrics, vol. 17, no. 1, pp. 13-23, 2006.

[5] G. S. Mudholkar and D. K. Srivastava, "Exponentiated Weibull family for analyzing bathtub failure-rate data," IEEE Transactions on Reliability, vol. 42, no. 2, pp. 299-302, 1993.

[6] S. K. Ashour and M. A. Eltehiwy, "Exponentiated power Lindley distribution," Journal of Advanced Research, vol. 6, no. 6, pp. 895-905, 2015.

[7] E. Gumbel, "Les valeurs extrêmes des distributions statistiques," Annales de l'Institut Henri Poincaré, vol. 5, no. 2, pp. 115-158, 1935.

[8] E. J. Gumbel, “The return period of flood flows," The Annals of Mathematical Statistics, vol. 12, pp. 163-190, 1941.

[9] E. J. Gumbel, Statistical Theory of Extreme Values and Some Practical Applications, vol. 33 of Applied Mathematics, U.S. Department of Commerce, National Bureau of Standards, ASIN B0007DSHG4, Gaithersburg, Md, USA, 1st edition, 1954.

[10] E. T. Lee and J. Wang, Statistical Methods for Survival Data Analysis, vol. 476, John Wiley \& Sons, New York, NY, USA, 2003.

[11] M. Abramowitz and I. A. Stegun, Handbook of Mathematical Functions, vol. 55 of Applied Mathematics Series, 1966.

[12] F. W. Olver, D. W. Lozier, R. F. Boisvert, and C. W. Clark, NIST Handbook of Mathematical Functions, US Department of Commerce, National Institute of Standards and Technology, 2010.

[13] K.-S. Song, "Rényi information, loglikelihood and an intrinsic distribution measure," Journal of Statistical Planning and Inference, vol. 93, no. 1-2, pp. 51-69, 2001.

[14] H. Akaike, "Information theory and an extension of the maximum likelihood principle," in Breakthroughs in Statistics, pp. 610-624, Springer, New York, NY, USA, 1992.

[15] N. Sugiura, "Further analysts of the data by akaike's information criterion and the finite corrections: further analysts of the data by akaike's," Communications in Statistics-Theory and Methods, vol. 7, no. 1, pp. 13-26, 1978.

[16] H. Bozdogan, "Model selection and Akaike's information criterion (AIC): the general theory and its analytical extensions," Psychometrika, vol. 52, no. 3, pp. 345-370, 1987. 
[17] G. Schwarz, "Estimating the dimension of a model," The Annals of Statistics, vol. 6, no. 2, pp. 461-464, 1978.

[18] E. J. Hannan and B. G. Quinn, "The determination of the order of an autoregression," Journal of the Royal Statistical Society B: Methodological, vol. 41, no. 2, pp. 190-195, 1979.

[19] A. Z. Afify, Z. M. Nofal, and N. S. Butt, "Transmuted complementary weibull geometric distribution," Pakistan Journal of Statistics and Operation Research, vol. 10, no. 4, pp. 435-454, 2014. 


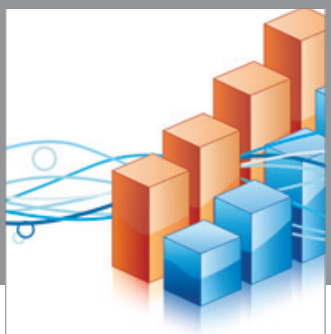

Advances in

Operations Research

vatem alat4

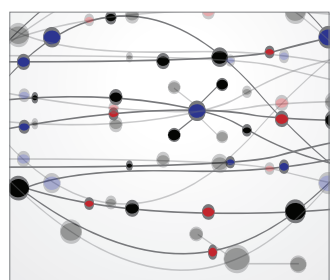

\section{The Scientific} World Journal
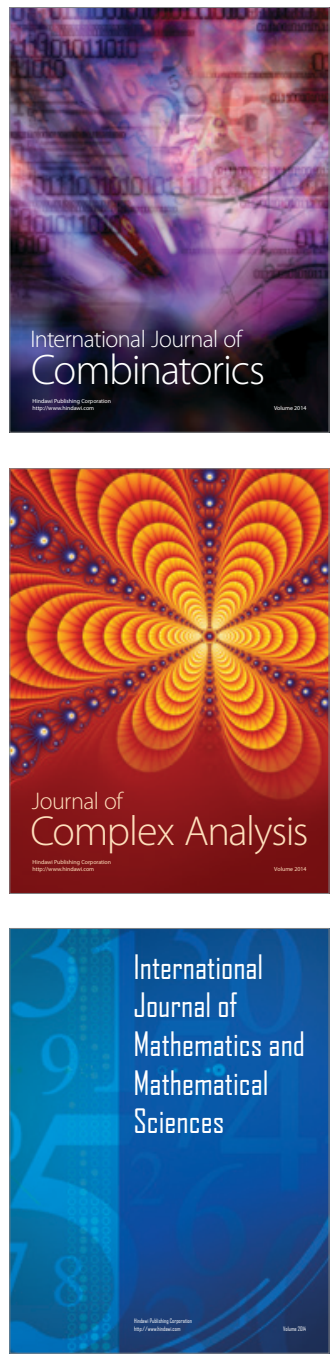
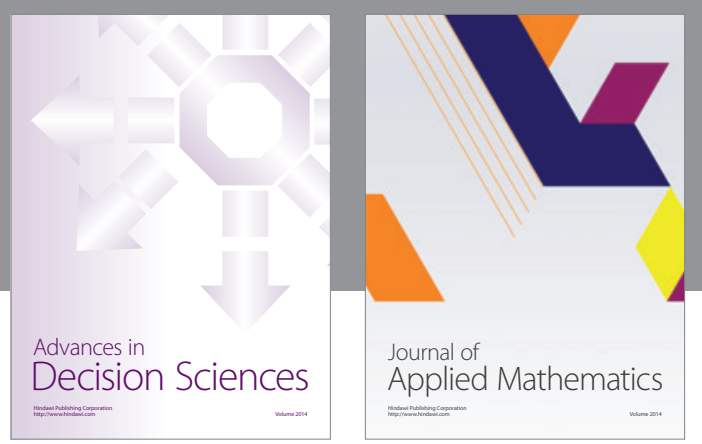

Algebra

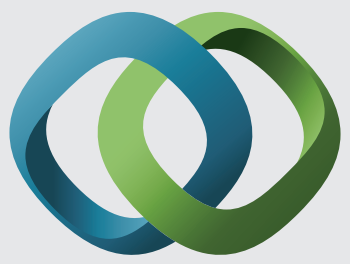

\section{Hindawi}

Submit your manuscripts at

http://www.hindawi.com
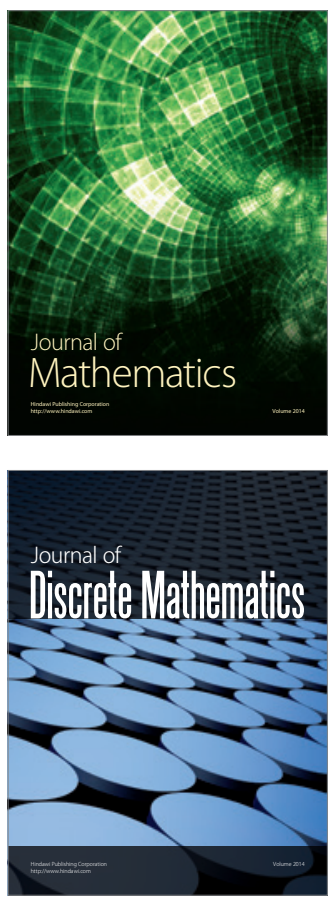

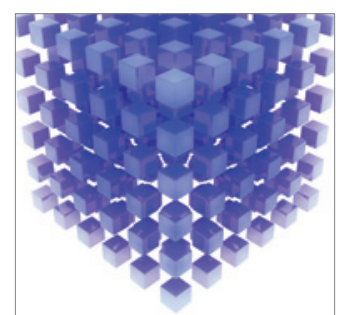

Mathematical Problems in Engineering
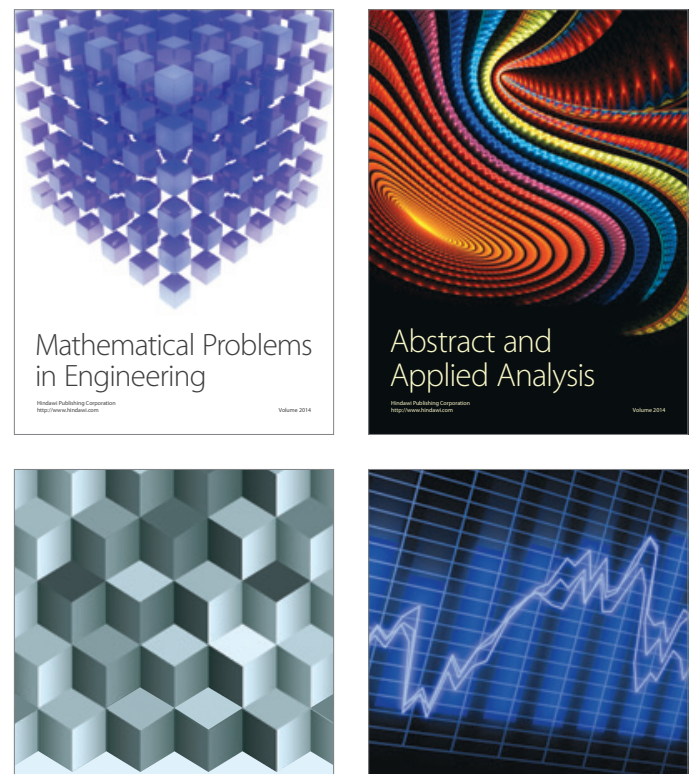

Journal of

Function Spaces

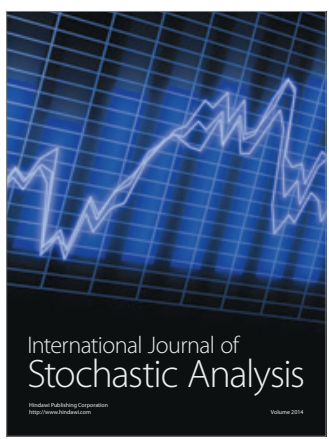

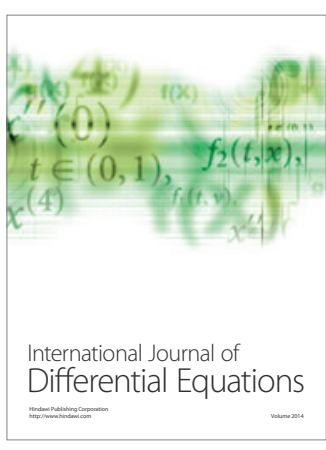
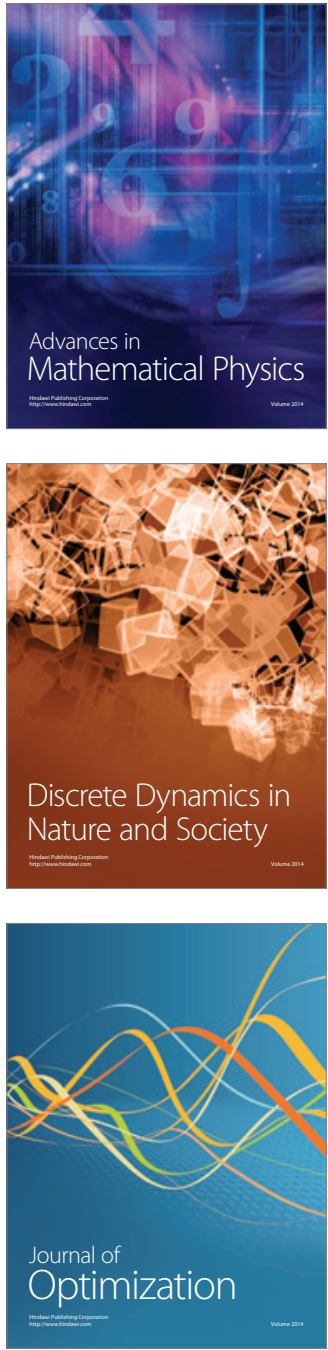